\title{
Pleural Monophasic Synovial Sarcoma
}

National Cancer Institute

\section{Source}

National Cancer Institute. Pleural Monophasic Synovial Sarcoma. NCI Thesaurus. Code C45697.

A monophasic synovial sarcoma arising in the pleural cavity. 